\title{
Postoperative changes in sagittal spinopelvic alignment in sitting position in adolescents with idiopathic thoracic scoliosis treated with posterior fusion: an initial analysis
}

\author{
*Weiguo Zhu, MD, Zhen Liu, MD, Shifu Sha, MD, Jing Guo, MD, Hongda Bao, MD, PhD, \\ Leilei Xu, MD, Yong Qiu, MD, and Zezhang Zhu, MD \\ Department of Spine Surgery, Drum Tower Hospital of Nanjing University Medical School, Nanjing, Jiangsu Province, China
}

\begin{abstract}
OBJECTIVE Previous studies have reported spinal straightening and pelvic retroversion when changing from erect to sitting posture in patients with adolescent idiopathic scoliosis (AIS), which were thought to be related to low-back pain after sitting for long periods. However, the sitting sagittal alignment after posterior spinal fusion has not been evaluated. This study aims to assess the influence of posterior fusion surgery upon sitting sagittal spinopelvic alignment in adolescents with idiopathic thoracic curves (thoracic AIS [T-AIS]).
\end{abstract}

METHODS A total of 44 T-AIS patients (30 Lenke I and 14 Lenke II) from the authors' center were included in this study. Preoperative and postoperative long-cassette lateral radiographs of the spine and pelvis were obtained with the patients in standing and sitting positions. Thoracic kyphosis (TK), lumbar lordosis (LL), pelvic incidence (PI), sacral slope (SS), and pelvic tilt (PT) were measured on standing and sitting lateral radiographs. Patients were divided into selective thoracic fusion (STF) and nonselective thoracic fusion (NSTF) groups.

RESULTS At baseline, TK, LL, and SS decreased by $27.5 \%, 42.1 \%$, and $31.1 \%$, respectively, from the standing to the sitting position, while PT increased by $193.6 \%$. After posterior spinal fusion, increased TK, LL, and SS and corresponding decreased PT were observed compared to baseline parameters in the sitting position. Comparison of postoperative sitting and standing values for the whole cohort showed that the mean LS and SS values were significantly lower in the sitting position (decreased by $14.0 \%$ and $13.9 \%$, respectively, compared to standing), whereas the mean PT value was significantly greater (increased by $39.0 \%$, compared to standing). Similar changes were also observed in the STF group: postoperatively the mean LL value was $15.6 \%$ lower in sitting than in standing, while the mean SS value was $11.5 \%$ lower. However, no obvious changes of the postoperative values in sitting were found in the NSTF group.

CONCLUSIONS Nonselective thoracic fusion surgery in T-AIS patients diminished spinal straightening and pelvic retroversion during sitting. Reducing distal fusion levels was of special value in not only saving more lumbar mobility, but also preserving the function of pelvic posterior rotation.

https://thejns.org/doi/abs/10.3171/2018.2.PEDS17687

KEYWORDS adolescent idiopathic thoracic scoliosis; sagittal spinopelvic alignment; sitting position; posterior spinal fusion; spine

$\mathrm{T}$ HE importance of sagittal spinopelvic alignment has been increasingly recognized with several radiographic parameters established for the assessment of the spine and pelvis. ${ }^{8,17}$ Numerous recent studies have focused on the standing sagittal spinal and pelvic profile. ${ }^{13,21-23}$ Normative data for these sagittal parameters in the standing position have been well established, and these data are currently used as the standards for sagittal plane correction of spinal deformity. ${ }^{12,25}$ Compared with the standing condition, however, little attention has been paid to the sitting sagittal profile. Previous studies had demonstrated that prolonged sitting was associated with the low-back pain and other lumbar disability. ${ }^{1,6}$ Therefore, full knowledge of the sagittal plane

ABBREVIATIONS AIS = adolescent idiopathic scoliosis; LIV = lowest instrumented vertebra; LL = lumbar lordosis; NSTF = nonselective thoracic fusion; PI = pelvic incidence; PT = pelvic tilt; SRS-22 = 22-item Scoliosis Research Society questionnaire; SS = sacral slope; STF = selective thoracic fusion; T-AIS = thoracic AIS; TK = thoracic kyphosis.

SUBMITTED December 4, 2017. ACCEPTED February 13, 2018.

INCLUDE WHEN CITING Published online May 4, 2018; DOI: 10.3171/2018.2.PEDS17687.

${ }^{*}$ W.Z. and Z.L. contributed equally to this work. 
of the spine and pelvis in the sitting position is also of critical importance.

In 2012, Endo et al. ${ }^{3}$ first documented the normal sagittal spinopelvic alignment in sitting in 50 healthy adults, which revealed decreased lumbar lordosis (LL) and sacral slope (SS), accompanied with increased pelvic tilt (PT) compared to standing sagittal alignment. The authors speculated that these results could help to understand the causes of low-back pain. These results were echoed by a recent study by Hey et al., ${ }^{5}$ demonstrating a reduction of thoracic kyphosis (TK), LL, and SS, accompanied by an increase of PT in sitting position in patients without spinal deformity. To reveal the changes of the sagittal profile of adolescent idiopathic scoliosis (AIS) in sitting, Vaughn and Schwend ${ }^{20}$ performed a similar study in patients with AIS and found that the spine was also significantly straightened, with decreases of TK, LL, and SS, in sitting position in primary scoliosis patients, and they recommended consideration of the changes of sagittal alignment in sitting when planning operative strategies.

At present, posterior spinal instrumentation and fusion is the main surgical management for the correction of AIS. ${ }^{14,19}$ The sagittal profile changes in standing position following posterior surgery have been well documented. ${ }^{7,16}$ However, to our best knowledge, the change of sagittal spinopelvic alignment after posterior spinal fusion in AIS patients in a seated position has not been documented, especially in the adjacent non-fusion levels. This study aimed to evaluate the sitting sagittal spinopelvic alignment of adolescent patients with thoracic scoliosis (T-AIS) after posterior correction surgery and to explore the influence of different fusion levels upon the sagittal alignment in sitting.

\section{Methods}

This study was approved by the ethics review board of the affiliated Drum Tower Hospital of Nanjing University.

\section{Subjects}

Forty-four cases involving patients with AIS treated with one-stage posterior spinal fusion surgery at our hospital between March 2013 and March 2015 were retrospectively reviewed. The inclusion criteria were as follows: 1) Lenke type I or II curve, ${ }^{9}$ 2) complete radiographic imaging at 6 months' follow-up, and 3) no evidence of hip or hamstring contractures. Patients with a history of any operations for spinal or pelvic trauma, infection, or tumor were excluded. There were 30 Lenke type I (6 1A, 12 1B, 12 1C) and 14 Lenke type II (4 2A, 6 2B, 4 2C) curves. All patients were treated by the same spinal surgeon with onestage correction surgery with posterior fusion and segmental instrumentation ranging from T-1 to L-4. Patients were further divided in 2 groups based on the level of the lowest instrumented vertebra (LIV): the selective thoracic fusion (STF) group (LIV T-12, L-1, or L-2; $\mathrm{n}=26$ ) and the nonselective thoracic fusion (NTSF) group (LIV L-3 or L-4; $\mathrm{n}=18$ ). In the STF group, the LIV was T-12 in 7 cases, L-1 in 17, and L-2 in 2. In NSTF group, the LIV was L-3 in 10 cases and L-4 in 8. Informed consent was obtained from all subjects.

\section{Surgical Technique}

A standard midline approach to the posterior elements was used with exposure via subperiosteal dissection. A complete pedicle screw system was used. The fusion level was basically decided according to Lenke classification. ${ }^{10}$ Nonselective thoracic fusion was performed in patients with Lenke $1 \mathrm{C}$ or $2 \mathrm{C}$, while selective thoracic fusion was performed in those with Lenke lumbar modifier A or B. Curve correction was achieved via translation technique, followed by moderate distraction on the concave side and compression on the convex side. Posterior fusion was accomplished with autograft and allograft. Neuromonitoring was used routinely throughout the operations.

\section{Radiographic and Clinical Evaluation}

Preoperative long-cassette lateral radiographs of the spine and pelvis were obtained in the fist-on-clavicle position $^{23}$ in standing and with the patient's hands on the clavicles $^{20}$ in sitting. In all cases, the above radiographs were obtained in standing and sitting positions 6 months after surgery. The sitting position was an upright posture on the same stool (without seat back) with the head and trunk vertical and feet on the ground; the lower legs were bent at about $90^{\circ}$ at the hips and knees. ${ }^{3}$ If the patient's feet could not touch the ground, a wooden step was put beneath their feet. The additional lateral radiograph in sitting was obtained as part of a general AIS data collection effort and was approved by the patients, their parents, and the institutional review board of our hospital. The following radiographic parameters were measured using Surgimap software (Nemaris, Inc.): TK (the angle between the superior endplate of T-5 and the inferior endplate of T-12), LL (the angle between the upper endplate of L-1 and the upper endplate of S-1), pelvic incidence (PI, the angle between the perpendicular to the sacral plate at its midpoint and the line connecting the point to the middle axis of the femoral heads), SS (the angle between the sacral plate and the horizontal plane), and PT (the angle between the line connecting the midpoint of the sacral plate to the axis of the femoral heads, and the gravity line) (Fig. 1).

All the patients were required to complete the 22-item Scoliosis Research Society questionnaire (SRS-22) before surgery and at 6 months' follow-up. There are 5 domains in the SRS-22, including function/activity, pain, selfperceived image, satisfaction with treatment, and mental health. The questions for each domain have 5 verbal response alternatives ranging from 1 to 5 . The value of 5 indicates the best outcome. Results of the SRS-22 are expressed using the mean value for each domain, which is calculated by dividing the total sum of the domain by the number of items answered.

All radiographic and clinical data collection was done by an independent observer (J.G.), who was not involved in the treatment of the patients. Radiographic parameters were measured twice, and the means of the 2 measurements were recorded.

\section{Statistical Analysis}

Values were expressed as mean \pm standard deviation. Statistical analysis was performed using the SPSS soft- 

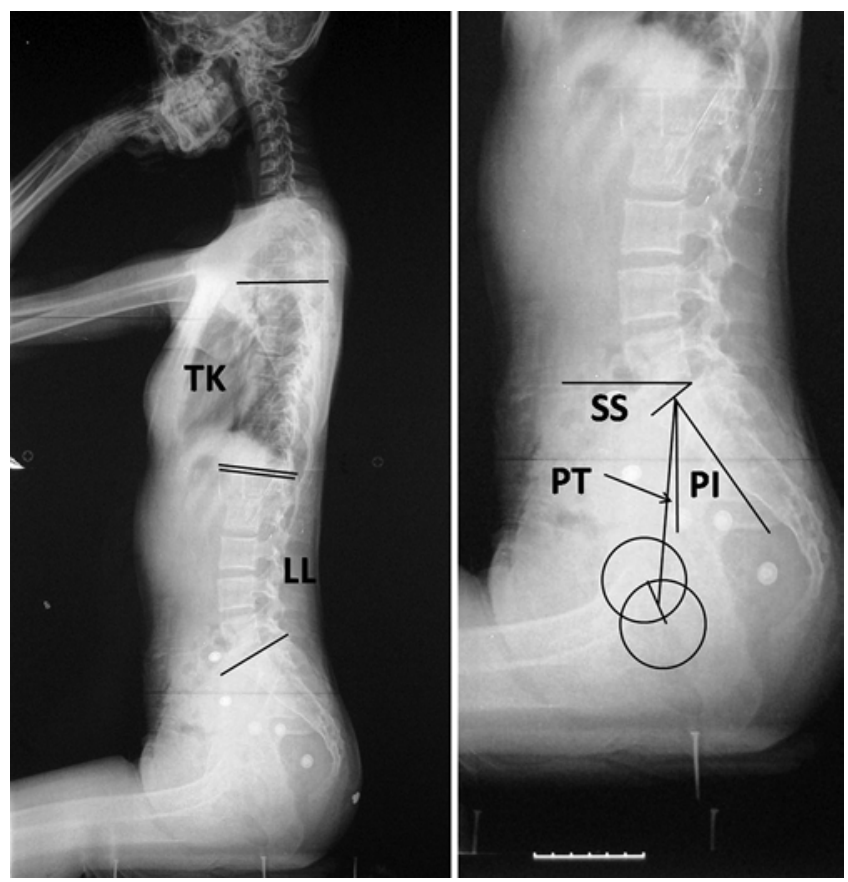

FIG. 1. Left: Spinal parameters: thoracic kyphosis (TK), lumbar lordosis (LL). Right: Pelvic parameters: pelvic incidence (PI), sacral slope (SS), and pelvic tilt (PT).

ware (v. 16.0, SPSS Inc.). Comparisons between pre- and postoperative radiographic values or between standing and sitting values were performed using the Student paired t-test for paired samples. In addition, radiographic and clinical data were compared between the STF group and the NSTF group with independent sample t-tests. Correlations between the radiographic changes from standing to sitting at the 6-month follow-up assessment and patient age were analyzed with the Pearson correlation coefficient. The Pearson analysis was also performed to assess the correlations between the radiographic changes and SRS-22 scores. Statistical significance was set at $\mathrm{p}$ $<0.05$.

\section{Results}

Five male and 39 female AIS patients were included in the study. Their average age was $13.5 \pm 1.4$ years (range 11-17 years). The mean value of the preoperative main thoracic Cobb angle was $52.2^{\circ} \pm 7.9^{\circ}$, and the postoperative value was $14.9^{\circ} \pm 5.7^{\circ}$ after surgical correction. Compared to the preoperative sitting measurements, TK, LL, and SS in sitting position increased significantly after surgery while PT decreased significantly (Table 1).

The sagittal spinopelvic alignment in standing and sitting before and after posterior fusion surgery was summarized in Table 2. Before surgery, TK, LL, and SS in sitting decreased, respectively, by $3.5^{\circ}(27.5 \%), 20.3^{\circ}(42.1 \%)$, and $11.7^{\circ}(31.1 \%)$ from the corresponding measurements in standing position, while PT in sitting increased by $11.8^{\circ}(193.6 \%)$. Six months after posterior fusion surgery, similar changes of these parameters except TK were also detected when seated ( $\mathrm{p}<0.05)$ : LL decreased by $4.5^{\circ}$ (9.7\%), SS decreased by $2.0^{\circ}(5.7 \%)$, and PT increased by $2.3^{\circ}(39.0 \%)$, with a lower magnitude of the parameter changes. To explore the influence of different fusion levels on the sitting sagittal profile, comparison between 3 different fusion strategies was performed. The STF $(n=26)$ and NSTF $(n=18)$ groups were comparable with respect to age, Risser grade, implant density, and sagittal profile (Table 3). As shown in Table 4, significant differences of the sagittal parameters between standing and sitting were observed before surgery in both groups. After surgery, straightening of the lumbar spine and retroversion of the pelvis were observed from standing to sitting in patients undergoing selective thoracic fusion (STF group) (Fig. 2). Specifically, LL was decreased by $6.9^{\circ}(15.6 \%)$, SS was decreased by $3.9^{\circ}(11.5 \%)$, and PT was increased by $4.1^{\circ}$ (43.2\%). In contrast, when spinal fusion was extended to L-3 or L-4 (NSTF group), no obvious change of sagittal spinopelvic parameters was observed from standing to sitting (Fig. 3).

As for the evaluation of quality of life, there were no significant differences between the STF group and the NSTF group in the 4 domains of function, pain, general function, and mental health preoperatively. Six months after surgery, the scores for function and pain in the STF group were significantly higher than those in the NSTF group (with higher scores indicating better function and less pain). The scores in the other domains were similar between the 2 groups (Table 5).

As for the correlation analysis, there was no signifi-

TABLE 1. Spinopelvic parameters: comparison of preoperative versus postoperative values obtained in standing and sitting positions

\begin{tabular}{|c|c|c|c|c|c|c|}
\hline \multirow[b]{2}{*}{ Parameters } & \multicolumn{2}{|c|}{ Standing } & \multirow[b]{2}{*}{$p$ Value } & \multicolumn{2}{|c|}{ Sitting } & \multirow[b]{2}{*}{$p$ Value } \\
\hline & Preop & Postop & & Preop & Postop & \\
\hline $\mathrm{TK}\left({ }^{\circ}\right)$ & $12.7 \pm 7.4$ & $17.3 \pm 3.8$ & $0.003^{*}$ & $9.2 \pm 6.8$ & $17.6 \pm 5.1$ & $<0.001^{*}$ \\
\hline $\operatorname{LL}\left({ }^{\circ}\right)$ & $48.3 \pm 11.3$ & $46.3 \pm 8.9$ & 0.793 & $28.0 \pm 16.5$ & $39.8 \pm 12.4$ & $<0.001^{*}$ \\
\hline $\mathrm{PI}\left({ }^{\circ}\right)$ & $43.6 \pm 10.1$ & $42.8 \pm 9.6$ & 0.806 & $43.8 \pm 11.1$ & $43.1 \pm 10.2$ & 0.625 \\
\hline PT $\left({ }^{\circ}\right)$ & $6.1 \pm 6.5$ & $5.9 \pm 5.8$ & 0.646 & $17.9 \pm 12.8$ & $10.2 \pm 7.9$ & $0.003^{*}$ \\
\hline $\operatorname{SS}\left({ }^{\circ}\right)$ & $37.5 \pm 9.0$ & $36.9 \pm 5.9$ & 0.388 & $25.8 \pm 13.4$ & $32.9 \pm 8.4$ & $0.016^{*}$ \\
\hline
\end{tabular}

$\mathrm{LL}=$ lumbar lordosis, $\mathrm{PI}$ = pelvic incidence, $\mathrm{PT}$ = pelvic tilt; $\mathrm{SS}$ = sacral slope; $\mathrm{TK}=$ thoracic kyphosis.

Data are means and standard deviations for the entire cohort.

* Significant difference $(p<0.05)$. 
TABLE 2. Spinopelvic parameters: comparison of values obtained in standing versus sitting position for preoperative and postoperative measurements

\begin{tabular}{|c|c|c|c|c|c|c|}
\hline \multirow[b]{2}{*}{ Parameters } & \multicolumn{2}{|c|}{ Preop } & \multirow[b]{2}{*}{$p$ Value } & \multicolumn{2}{|c|}{ Postop } & \multirow[b]{2}{*}{ p Value } \\
\hline & Standing & Sitting & & Standing & Sitting & \\
\hline $\operatorname{TK}\left({ }^{\circ}\right)$ & $12.7 \pm 7.4$ & $9.2 \pm 6.8$ & $0.001^{*}$ & $17.3 \pm 3.8$ & $17.6 \pm 5.1$ & 0.708 \\
\hline $\operatorname{LL}\left({ }^{\circ}\right)$ & $48.3 \pm 11.3$ & $28.0 \pm 16.5$ & $<0.001^{*}$ & $46.3 \pm 8.9$ & $39.8 \pm 12.4$ & $0.007^{*}$ \\
\hline $\mathrm{PI}\left({ }^{\circ}\right)$ & $43.6 \pm 10.1$ & $43.8 \pm 11.1$ & 0.772 & $42.8 \pm 9.6$ & $43.1 \pm 10.2$ & 0.415 \\
\hline $\mathrm{PT}\left({ }^{\circ}\right)$ & $6.1 \pm 6.5$ & $17.9 \pm 12.8$ & $0.001^{*}$ & $5.9 \pm 5.8$ & $10.2 \pm 7.9$ & $0.037^{*}$ \\
\hline $\operatorname{SS}\left({ }^{\circ}\right)$ & $37.5 \pm 9.0$ & $25.8 \pm 13.4$ & $0.001^{*}$ & $36.9 \pm 5.9$ & $32.9 \pm 8.4$ & $0.024^{*}$ \\
\hline
\end{tabular}

Data are means and standard deviations for the entire cohort.

* Significant difference $(p<0.05)$.

cant relationship between age and radiographic changes at 6-month follow-up, in terms of LL $(r=0.102, p=0.441)$, PT $(r=0.096, p=0.517)$, and SS $(r=0.104, p=0.363)$. However, the function scores in the SRS-22 assessment were statistically correlated with LL $(r=0.279, p=0.037)$ and SS $(r=0.203, p=0.044)$. No other significant correlations were found between the radiographic changes and the quality-of-life outcomes.

\section{Discussion}

Sagittal spinal alignment, highly correlated with the quality of daily life, can be influenced by several factors, such as aging, ${ }^{12}$ wearing high-heeled shoes,${ }^{2}$ and position. ${ }^{11}$ It has been revealed that sitting posture can significantly straighten the spine and posteriorly rotate the pelvis in both normal subjects and patients with AIS. ${ }^{3,5,20}$ In sitting, the innominate bone moves to the posterior point of the hip axis, which posteriorly rotates the pelvis in the sagittal plane. ${ }^{3}$ Retroversion of the pelvis subsequently results in the decrease of the tilt of SS. With the correlation between the lumbar spine and sacrum revealed by biomechanical studies ${ }^{15,18}$ change of LL could occur as a result of adaptation for decreased SS. The results of our study showed a corresponding change of preoperative spinopelvic align-

TABLE 3. Comparison of clinical and radiographic data between the STF and NSTF groups

\begin{tabular}{lccc}
\hline \multirow{2}{*}{ Variable } & \multicolumn{2}{c}{ Group } & \\
\cline { 2 - 3 } & \multicolumn{1}{c}{ STF } & NSTF & p Value \\
\hline No. of pts & $13.8 \pm 1.7$ & $13.0 \pm 0.5$ & 0.246 \\
\hline Age & $51.6 \pm 4.5$ & $57.5 \pm 10.0$ & $0.012^{*}$ \\
\hline Cobb angle $\left(^{\circ}\right)$ & $2.0 \pm 1.0$ & $2.1 \pm 1.0$ & 0.27 \\
\hline Risser grade & $15.4 \pm 1.2$ & $16.2 \pm 1.8$ & 0.777 \\
\hline Implant density & $11.5 \pm 8.3$ & $13.9 \pm 5.5$ & 0.146 \\
\hline TK $\left(^{\circ}\right)$ & $49.9 \pm 9.6$ & $45.9 \pm 14.1$ & 0.109 \\
\hline LL $\left(^{\circ}\right)$ & $43.9 \pm 8.1$ & $43.4 \pm 11.4$ & 0.383 \\
\hline $\mathrm{PI}\left({ }^{\circ}\right)$ & $6.0 \pm 6.0$ & $6.1 \pm 6.7$ & 0.275 \\
\hline $\mathrm{PT}\left(^{\circ}\right)$ & $38.9 \pm 7.8$ & $37.3 \pm 10.9$ & 0.205 \\
\hline SS $\left(^{\circ}\right)$ & & &
\end{tabular}

$\mathrm{Pt}=$ patient.

Data are means and standard deviations unless otherwise specified.

* Significant difference $(p<0.05)$. ment in sitting, with decreases in TK (27.5\%), LL (42.1\%), and SS (31.1\%) and increase in PT (193.6\%), which was similar to the results reported by Vaughn and Schwend ${ }^{20}$ in their study of 26 children with idiopathic scoliosis. They reported a decreased TK by $17.1 \%$, a decreased LL by $46.2 \%$, a decreased SS by $47.2 \%$, and an increased PT by $280.7 \%$ in sitting compared with standing. However, to the best of our knowledge, the sitting sagittal profile of the spine and pelvis after posterior spinal fusion has not been previously reported.

In this study we evaluated the sagittal spinopelvic alignment in sitting position after posterior fusion in TAIS patients and found that the changes of lumbar spinal straightening (LL 14.0\%, SS 13.9\%) and pelvic posterior rotation also existed when patients were seated after posterior fusion (Table 1). Due to the long instrumentation in the thoracic spine for deformity correction, changes of TK after surgery in sitting position should then be nonsignificant. In addition, compared with the preoperative changes of the lumbar and pelvis (LL 42.1\%, SS 33.1\%) from standing to sitting, the magnitude of the postoperative changes was lower (Table 2). In our opinion, fusion induced the decrease of the whole lumbar mobility, which limited the changes of lumbar segment from standing to sitting after surgery. According to the reciprocal segments' influence on the lumbar and sacrum, ${ }^{18}$ the less range of lumbar mobility restricted the retroversion of pelvis. Therefore, the decrease of LL and SS and increase of PT after operation from standing to sitting was limited, leading to the larger LL and SS and the smaller PT than preoperative values in sitting position (Fig. 2). Our finding was in accordance with the above conclusion: compared with the preoperative sitting sagittal profile, TK (151.3\%), LL (47.5\%), and SS $(24.2 \%)$ in sitting after surgery were significantly increased and PT (52.9\%) was significantly decreased.

In thoracic scoliosis surgery, how to determine an optimal distal fusion level is still controversial. ${ }^{4}$ Our results further revealed the special value in preserving the function of pelvic retroversion with selective fusion in lumbar segments. In our series, the pelvis was posteriorly rotated $11.5 \%$ in the STF group when the patients were seated, while no significant alteration of pelvic orientation was observed from standing to sitting when the distal fusion level was extended to L-3 or L-4 in the NSTF group (Table 4). In the lumbar spine, the mobility was not evenly distributed from L-1 to the sacrum, with most of the mobility 
TABLE 4. Spinopelvic parameters: comparison of values obtained standing versus sitting for preoperative and postoperative measurements in the 2 groups

\begin{tabular}{|c|c|c|c|c|c|c|}
\hline \multirow[b]{2}{*}{ Group \& Parameter } & \multicolumn{2}{|c|}{ Preop } & \multirow[b]{2}{*}{ p Value } & \multicolumn{2}{|c|}{ Postop } & \multirow[b]{2}{*}{$p$ Value } \\
\hline & Standing & Sitting & & Standing & Sitting & \\
\hline \multicolumn{7}{|l|}{ STF group $(n=26)$} \\
\hline $\mathrm{TK}\left({ }^{\circ}\right)$ & $11.5 \pm 8.3$ & $5.9 \pm 6.0$ & $0.006^{*}$ & $16.2 \pm 3.5$ & $16.0 \pm 4.2$ & 0.858 \\
\hline $\operatorname{LL}\left({ }^{\circ}\right)$ & $49.9 \pm 9.6$ & $23.0 \pm 17.3$ & $<0.001^{*}$ & $44.3 \pm 9.6$ & $37.4 \pm 12.8$ & $0.003^{*}$ \\
\hline $\mathrm{PI}\left({ }^{\circ}\right)$ & $43.9 \pm 8.1$ & $44.5 \pm 9.4$ & 0.426 & $43.4 \pm 8.1$ & $42.6 \pm 8.6$ & 0.602 \\
\hline $\mathrm{PT}\left({ }^{\circ}\right)$ & $6.0 \pm 6.0$ & $22.1 \pm 12.9$ & $<0.001^{*}$ & $7.5 \pm 6.7$ & $13.6 \pm 7.1$ & $0.013^{*}$ \\
\hline SS $\left(^{\circ}\right)$ & $38.9 \pm 7.8$ & $22.5 \pm 14.0$ & $0.001^{*}$ & $35.9 \pm 6.5$ & $29.0 \pm 8.3$ & $0.010^{*}$ \\
\hline \multicolumn{7}{|l|}{ NSTF group $(n=18)$} \\
\hline $\mathrm{TK}\left({ }^{\circ}\right)$ & $13.9 \pm 5.5$ & $9.4 \pm 7.2$ & $0.015^{*}$ & $19.3 \pm 3.7$ & $20.5 \pm 5.6$ & 0.393 \\
\hline $\operatorname{LL}\left({ }^{\circ}\right)$ & $45.9 \pm 14.1$ & $25.9 \pm 12.5$ & $0.005^{*}$ & $50.0 \pm 6.4$ & $48.7 \pm 6.5$ & 0.723 \\
\hline $\mathrm{PI}\left({ }^{\circ}\right)$ & $43.4 \pm 11.4$ & $42.9 \pm 11.6$ & 0.508 & $42.2 \pm 11.2$ & $43.5 \pm 11.9$ & 0.465 \\
\hline $\mathrm{PT}\left({ }^{\circ}\right)$ & $6.1 \pm 6.7$ & $10.7 \pm 10.0$ & $0.014^{*}$ & $3.7 \pm 6.5$ & $4.2 \pm 7.4$ & 0.158 \\
\hline SS $\left(^{\circ}\right)$ & $37.3 \pm 10.9$ & $31.1 \pm 11.6$ & $0.007^{*}$ & $38.8 \pm 4.5$ & $37.6 \pm 5.9$ & 0.791 \\
\hline
\end{tabular}

Data are means and standard deviations.

* Significant difference $(p<0.05)$.

in the lower lumbar spine. ${ }^{15}$ Vaughn and Schwend ${ }^{20}$ also demonstrated that the greatest motion of the segmental LL (measured by disc angles) from standing to sitting was at the lower lumbar segments. When the distal fusion level extended to the lower lumbar segments, most of the lumbar mobility was restricted, which eliminates the pelvic retroversion function (Fig. 3). Therefore, STF played an important role in maintaining the lumbopelvic mobility, which was closely related to quality of life according to our clinical assessment. As for the SRS-22 evaluation of function, the mean score for the STF group was significantly better than that of the NSTF group 6 months after surgery ( $4.1 \pm 0.85$ vs $3.3 \pm 0.73, p=0.019$, Table 5). Moreover, the function scores were statistically correlated with the variation in LL $(r=0.279, p=0.037)$ and SS $(r=$ $0.203, p=0.044)$. We deemed that the considerable ability of lumbopelvic alteration preserved by STF allowed the freedom of movement, which contributed to better quality of life.

In addition, kinetics and morphological studies ${ }^{24}$ have
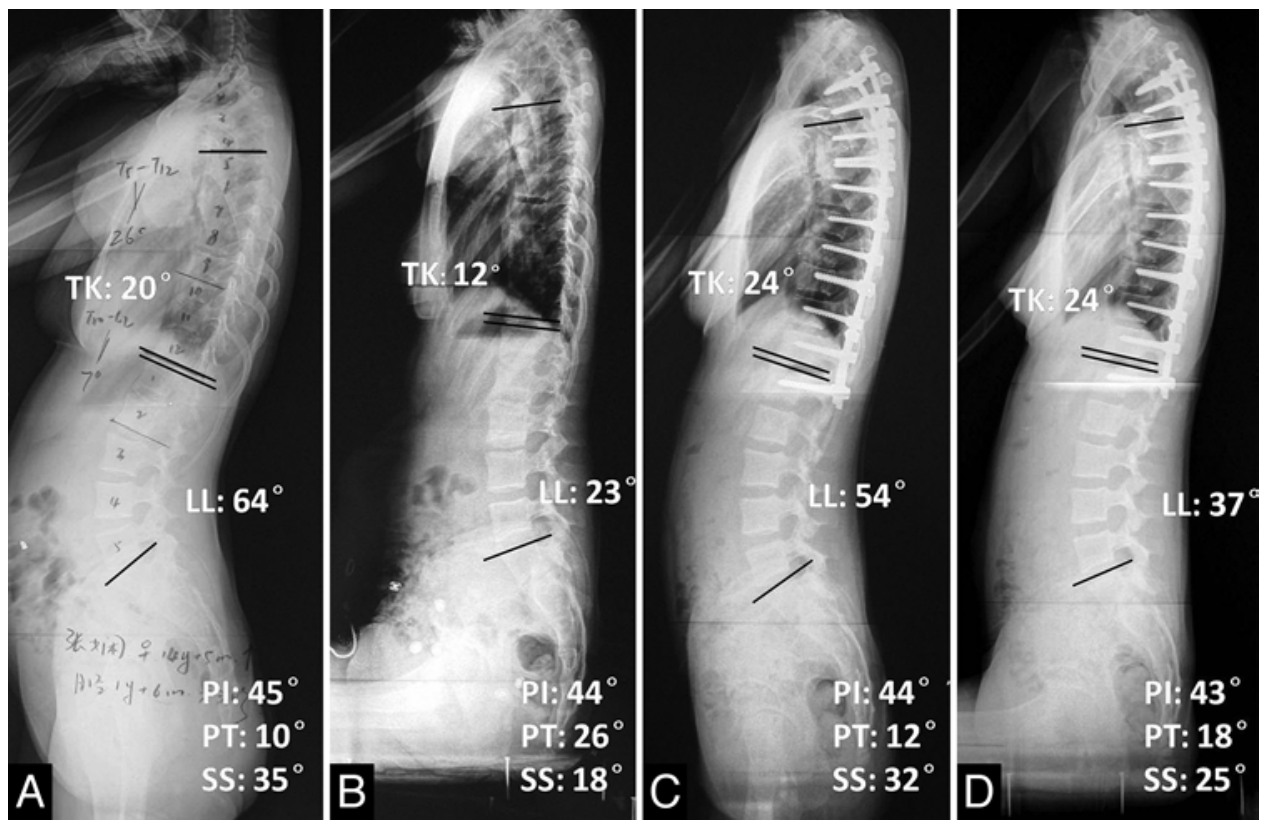

FIG. 2. Preoperative and postoperative radiographs obtained in a 15 -year-old female patient who underwent a selective posterior spinal fusion from T-3 to L-1. A and B: Preoperative values of the parameters in standing and sitting. In sitting, the spine was straightened and the pelvis was posteriorly rotated, with decreases in TK, LL, and SS and an increase in PT. C and D: Postoperative values of the parameters in standing and sitting. The lumbar spine was straightened in sitting, and the pelvis was posteriorly rotated, with decreases in LL and SS and an increase in PT. 

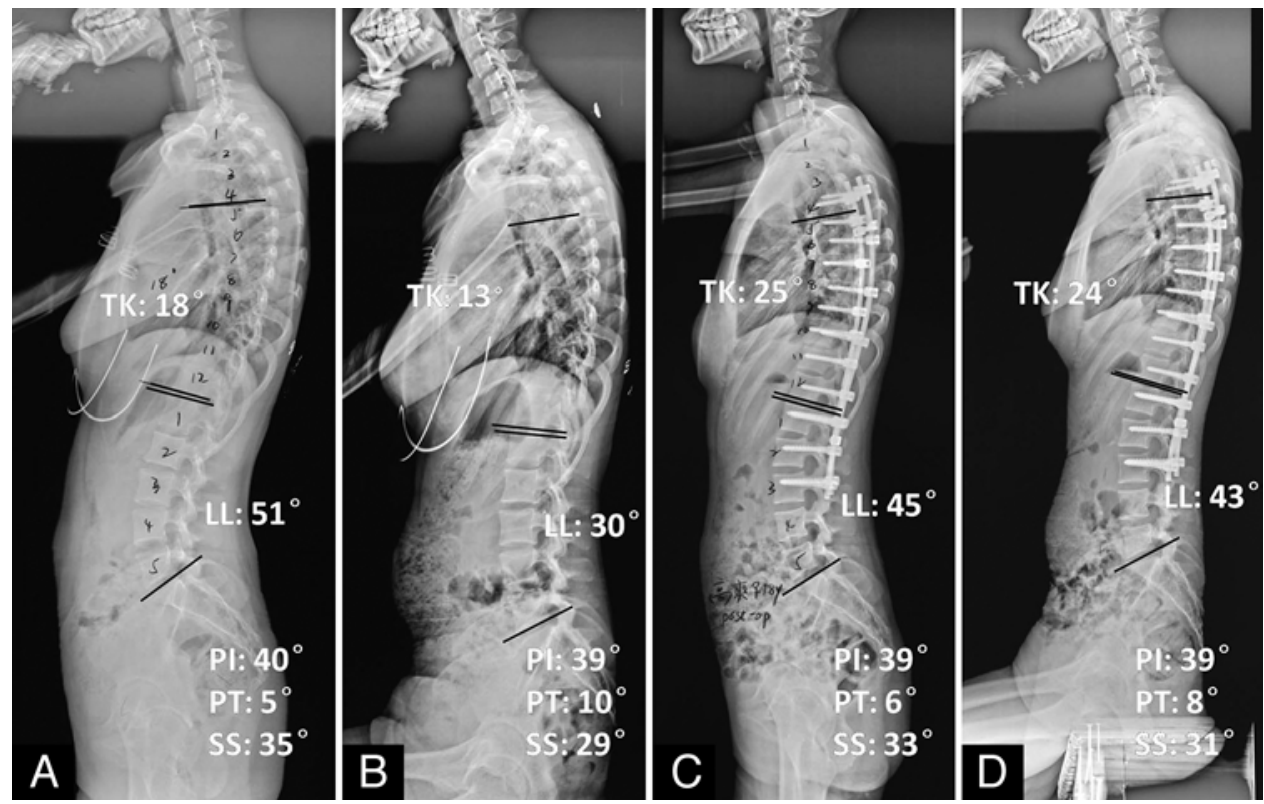

FIG. 3. Preoperative and postoperative radiographs obtained in an 18-year-old female patient who underwent a nonselective posterior spinal fusion from T-4 to L-3. A and B: Preoperative values of the parameters in standing (A) and sitting (B). In sitting, the spine was straightened and the pelvis was posteriorly rotated with decreases in TK, LL, and SS and an increase of PT. C and D: Postoperative values of the parameters in standing (C) and sitting (D). The sagittal spinopelvic alignment did not change significantly from standing to sitting.

demonstrated that pelvic retroversion decreases psoas muscle tension when seated. In this regard, the limited pelvic retroversion in patients with nonselective fusion would increase psoas muscle tension in sitting, which would potentially contribute to low-back pain after posterior spinal fusion. This theory was supported by our SRS-22 result, with a lower pain score (indicating greater pain) in the NSTF compared with the STF group $(4.2 \pm 0.74$ vs $3.5 \pm$ $0.61, \mathrm{p}=0.031$, Table 5). Our findings would help in better understanding the mechanism of low-back pain in nonselective fusion patients.

Our study demonstrated a consistent change from stand-

TABLE 5. SRS-22 results: comparison of results in the STF and NSTF groups

\begin{tabular}{cccc}
\hline $\begin{array}{c}\text { Time Point } \\
\text { \& Domain }\end{array}$ & $\begin{array}{c}\text { STF Group } \\
(\mathrm{n}=26)\end{array}$ & $\begin{array}{c}\text { NSTF Group } \\
(\mathrm{n}=18)\end{array}$ & $\begin{array}{c}\mathrm{p} \\
\text { Value }\end{array}$ \\
\hline Preop & & & \\
\hline Function & $4.6 \pm 0.45$ & $4.5 \pm 0.52$ & 0.516 \\
\hline Pain & $4.5 \pm 0.48$ & $4.4 \pm 0.49$ & 0.581 \\
\hline Self-image & $3.4 \pm 0.71$ & $3.4 \pm 0.73$ & 0.766 \\
\hline Mental health & $4.1 \pm 0.50$ & $3.9 \pm 0.62$ & 0.192 \\
\hline 6 mos postop & & & \\
\hline Function & $4.1 \pm 0.85$ & $3.3 \pm 0.73$ & $0.019^{*}$ \\
\hline Pain & $4.2 \pm 0.74$ & $3.5 \pm 0.61$ & $0.031^{*}$ \\
\hline Self-image & $4.4 \pm 0.66$ & $4.3 \pm 0.87$ & 0.504 \\
\hline Satisfaction & $4.3 \pm 0.72$ & $4.0 \pm 0.75$ & 0.171 \\
\hline Mental health & $4.0 \pm 0.43$ & $4.0 \pm 0.69$ & 0.690 \\
\hline
\end{tabular}

* Significant difference $(p<0.05)$. ing to sitting, with the spine straightening and the pelvic retroversion in T-AIS patients before and after posterior fusion. Although the sagittal pelvic alignment changed in sitting, our measurements remained consistent with the equation PI $=$ PT + SS. In addition, increasing the lumbar fusion segments would restrict the posterior rotation of the pelvis, which was unfavorable to the quality of life in terms of function and pain based on our clinical outcomes.

There are some limitations of this study. First, the number of subjects is relatively small. Second, because there were only 5 males in the whole cohort, it is not powered to investigate sex-related changes in spinopelvic alignment. Third, the magnitude and flexibility of the coronal deformity may have an effect on the sagittal alignment; therefore, the loss of pelvic retroversion function after surgery in the NSTF group may be partly due to the larger magnitude of the main curve and Lenke lumbar modifiers. Fourth, radiograph obtained 6 months after surgery may not reflect a patient's ultimate alignment in the sitting position. Therefore, further investigation with longer follow-up based on the present initial analysis is needed to explore the natural history of sagittal spinopelvic alignment in sitting after correction surgery. However, we believe the present results could help in choosing the optimal distal fusion level in scoliosis surgery and contribute to the understanding of low-back pain in patients with nonselective spinal fusion.

\section{Conclusions}

Sitting position significantly straightens the spine and rotates the pelvis posteriorly, with decreases of TK, LL, and SS and increases of PT. Posterior spinal fusion lim- 
its the changes of the sagittal spinopelvic alignment from standing to sitting in adolescents with idiopathic thoracic scoliosis. Reducing distal fusion levels was of special value not only in preserving more lumbar mobility but also in protecting the function of pelvic retroversion. These findings should be taken into consideration when planning surgical strategy.

\section{Acknowledgments}

National Natural Science Foundation of China (grant no. 81171672) and the Development Project of Nanjing Science and Technology Commission and Foundation (grant no. 201402028) funds were received in support of this work.

\section{References}

1. Billy GG, Lemieux SK, Chow MX: Changes in lumbar disk morphology associated with prolonged sitting assessed by magnetic resonance imaging. PM R 6:790-795, 2014

2. Dai M, Li X, Zhou X, Hu Y, Luo Q, Zhou S: High-heeled-related alterations in the static sagittal profile of the spino-pelvic structure in young women. Eur Spine J 24:1274-1281, 2015

3. Endo K, Suzuki H, Nishimura H, Tanaka H, Shishido T, Yamamoto K: Sagittal lumbar and pelvic alignment in the standing and sitting positions. J Orthop Sci 17:682-686, 2012

4. Erdemir C, Musaoğlu R, Selek Ö, Gök Ü, Şarlak AY: Distal fusion level selection in Lenke 1A curves according to axial plane analyses. Spine (Phila Pa 1976) J 15:2378-2384, 2015

5. Hey HWD, Teo AQA, Tan KA, Ng LWN, Lau LL, Liu KG, et al: How the spine differs in standing and in sitting-important considerations for correction of spinal deformity. Spine $\mathbf{J}$ 17:799-806, 2017

6. Inoue G, Miyagi M, Uchida K, Ishikawa T, Kamoda H, Eguchi Y, et al: The prevalence and characteristics of low back pain among sitting workers in a Japanese manufacturing company. J Orthop Sci 20:23-30, 2015

7. La Maida GA, Zottarelli L, Mineo GV, Misaggi B: Sagittal balance in adolescent idiopathic scoliosis: radiographic study of spino-pelvic compensation after surgery. Eur Spine J 22 (Suppl 6):S859-S867, 2013

8. Legaye J, Duval-Beaupère G, Hecquet J, Marty C: Pelvic incidence: a fundamental pelvic parameter for three-dimensional regulation of spinal sagittal curves. Eur Spine J 7:99-103, 1998

9. Lenke LG, Betz RR, Harms J, Bridwell KH, Clements DH, Lowe TG, et al: Adolescent idiopathic scoliosis: a new classification to determine extent of spinal arthrodesis. J Bone Joint Surg Am 83-A:1169-1181, 2001

10. Lenke LG, Edwards CC II, Bridwell KH: The Lenke classification of adolescent idiopathic scoliosis: how it organizes curve patterns as a template to perform selective fusions of the spine. Spine (Phila Pa 1976) 28:S199-S207, 2003

11. Lord MJ, Small JM, Dinsay JM, Watkins RG: Lumbar lordosis. Effects of sitting and standing. Spine (Phila Pa 1976) 22:2571-2574, 1997

12. Mac-Thiong JM, Berthonnaud E, Dimar JR II, Betz RR, Labelle H: Sagittal alignment of the spine and pelvis during growth. Spine (Phila Pa 1976) 29:1642-1647, 2004

13. Mac-Thiong JM, Labelle H, Berthonnaud E, Betz RR, Roussouly P: Sagittal spinopelvic balance in normal children and adolescents. Eur Spine J 16:227-234, 2007
14. Mladenov KV, Vaeterlein C, Stuecker R: Selective posterior thoracic fusion by means of direct vertebral derotation in adolescent idiopathic scoliosis: effects on the sagittal alignment. Eur Spine J 20:1114-1117, 2011

15. Roussouly P, Berthonnaud E, Dimnet J: [Geometrical and mechanical analysis of lumbar lordosis in an asymptomatic population: proposed classification.] Rev Chir Orthop Reparatrice Appar Mot 89:632-639, 2003 (Fr)

16. Roussouly P, Labelle H, Rouissi J, Bodin A: Pre- and postoperative sagittal balance in idiopathic scoliosis: a comparison over the ages of two cohorts of 132 adolescents and 52 adults. Eur Spine J 22 (Suppl 2):S203-S215, 2013

17. Roussouly P, Nnadi C: Sagittal plane deformity: an overview of interpretation and management. Eur Spine J 19:18241836,2010

18. Roussouly P, Pinheiro-Franco JL: Sagittal parameters of the spine: biomechanical approach. Eur Spine J 20 (Suppl 5):578-585, 2011

19. Suk SI, Lee SM, Chung ER, Kim JH, Kim SS: Selective thoracic fusion with segmental pedicle screw fixation in the treatment of thoracic idiopathic scoliosis: more than 5-year follow-up. Spine (Phila Pa 1976) 30:1602-1609, 2005

20. Vaughn JJ, Schwend RM: Sitting sagittal balance is different from standing balance in children with scoliosis. J Pediatr Orthop 34:202-207, 2014

21. Vedantam R, Lenke LG, Keeney JA, Bridwell KH: Comparison of standing sagittal spinal alignment in asymptomatic adolescents and adults. Spine (Phila Pa 1976) 23:211-215, 1998

22. Vialle R, Levassor N, Rillardon L, Templier A, Skalli W, Guigui P: Radiographic analysis of the sagittal alignment and balance of the spine in asymptomatic subjects. J Bone Joint Surg Am 87:260-267, 2005

23. Yong Q, Zhen L, Zezhang Z, Bangping Q, Feng Z, Tao W, et al: Comparison of sagittal spinopelvic alignment in Chinese adolescents with and without idiopathic thoracic scoliosis. Spine (Phila Pa 1976) 37:E714-E720, 2012

24. Yoshio M, Murakami G, Sato T, Sato S, Noriyasu S: The function of the psoas major muscle: passive kinetics and morphological studies using donated cadavers. J Orthop Sci 7:199-207, 2002

25. Zhu Z, Xu L, Zhu F, Jiang L, Wang Z, Liu Z, et al: Sagittal alignment of spine and pelvis in asymptomatic adults: norms in Chinese populations. Spine (Phila Pa 1976) 39:E1-E6, 2014

\section{Disclosures}

The authors report no conflict of interest concerning the materials or methods used in this study or the findings specified in this paper.

\section{Author Contributions}

Conception and design: Z Zhu, W Zhu, Liu. Acquisition of data: Guo. Analysis and interpretation of data: Guo, Xu. Drafting the article: W Zhu. Critically revising the article: W Zhu, Liu, Sha. Reviewed submitted version of manuscript: Bao, Qiu. Approved the final version of the manuscript on behalf of all authors: Z Zhu.

\section{Correspondence}

Zezhang Zhu: Drum Tower Hospital of Nanjing University Medical School, Nanjing, Jiangsu Province, China. njzhuzezhang@163.com. 